

\title{
Profiling BCI users based on contralateral activity to improve kinesthetic motor imagery detection
}

Sébastien Rimbert, Cecilia Lindig-León, Laurent Bougrain

\section{To cite this version:}

Sébastien Rimbert, Cecilia Lindig-León, Laurent Bougrain. Profiling BCI users based on contralateral activity to improve kinesthetic motor imagery detection. 8th Internationl IEEE EMBS Conference On Neural Engineering, May 2017, Shanghai, China. hal-01484636

\author{
HAL Id: hal-01484636 \\ https://hal.science/hal-01484636
}

Submitted on 7 Mar 2017

HAL is a multi-disciplinary open access archive for the deposit and dissemination of scientific research documents, whether they are published or not. The documents may come from teaching and research institutions in France or abroad, or from public or private research centers.
L'archive ouverte pluridisciplinaire HAL, est destinée au dépôt et à la diffusion de documents scientifiques de niveau recherche, publiés ou non, émanant des établissements d'enseignement et de recherche français ou étrangers, des laboratoires publics ou privés. 


\title{
Profiling BCI users based on contralateral activity to improve kinesthetic motor imagery detection
}

\author{
Sébastien Rimbert ${ }^{1,2,3}$, Cecilia Lindig-León ${ }^{1,2,3}$ and Laurent Bougrain ${ }^{2,1,3}$
}

\begin{abstract}
Kinesthetic motor imagery (KMI) tasks induce brain oscillations over specific regions of the primary motor cortex within the contralateral hemisphere of the body part involved in the process. This activity can be measured through the analysis of electroencephalographic (EEG) recordings and is particularly interesting for Brain-Computer Interface (BCI) applications. The most common approach for classification consists of analyzing the signal during the course of the motor task within a frequency range including the alpha band, which attempts to detect the Event-Related Desynchronization (ERD) characteristics of the physiological phenomenon. However, to discriminate right-hand KMI and left-hand KMI, this scheme can lead to poor results on subjects for which the lateralization is not significant enough. To solve this problem, we propose that the signal be analyzed at the end of the motor imagery within a higher frequency range, which contains the EventRelated Synchronization (ERS). This study found that 6 out of 15 subjects have a higher classification rate after the KMI than during the KMI, due to a higher lateralization during this period. Thus, for this population we can obtain a significant improvement of $13 \%$ in classification taking into account the users lateralization profile.
\end{abstract}

\section{INTRODUCTION}

Brain-Computer interfaces (BCI) allow users to interact with a system using brain activity modulation mainly in electroencephalographic (EEG) signals [1]. One major interaction mode is based on the detection of modulations of sensorimotor rhythms during a kinesthetic motor imagery (KMI), i.e, the ability to imagine performing a movement without executing it [2], [3]. More precisely, alpha (7-13 $\mathrm{Hz})$ and beta rhythms $(15-25 \mathrm{~Hz})$ modulations can be observed measuring Event-Related Desynchronization (ERD) or Synchronization (ERS). In particular, before and during an imagined movement, there is a gradual decrease of power, mainly in the alpha band. Furthermore, after the end of the motor imagery, in the beta band, there is an increase of power called ERS or post-movement beta rebound [4].

A KMI generates an activity over specific regions of the primary motor cortex within the contralateral hemisphere of the body part used in the process [5]. Some BCIs are based on this contralateral activation to differentiate the cerebral activity generated by a right-hand KMI from a left-hand KMI [6]. Usually, the modulation corresponding to a user interaction is scanned in specific frequency bands such as Alpha, Beta or Alpha+Beta $(8-30 \mathrm{~Hz})$. This activity is mainly

\footnotetext{
*This work has been supported by the Inria project BCI LIFT

${ }^{1}$ Neurosys team, Inria, Villers-lès-Nancy, F-54600, France

${ }^{2}$ Artificial Intelligence and Complex Systems, Université de Lorraine, LORIA, UMR 7503, Vandœuvre-lès-Nancy, F-54506

${ }^{3}$ Neurosys team CNRS, LORIA, UMR 7503, Vandœuvre-lès-Nancy, F54506
}

observed, during the KMI in the $8-30 \mathrm{~Hz}$ band, which merge alpha and beta bands, or after the KMI in the beta band [7].

Detection rates for these two KMI tasks vary with subjects and could be improved. Indeed, between 15\% and 30\% of the users are considered as BCI-illiterate and cannot control a BCI [8]. In this article, we suggest that some of the so-called BCI-illiterate subjects have poor performance due to poor lateralization during the KMI task. Several studies showed activity only in the contralateral area [9] for a KMI, but other studies showed that ERD and ERS are also in the ipsilateral area [10] and could be a problem for BCI classification.

According to our knowledge, no studies compare the classifier accuracy based on signals observed during the KMI versus after the KMI. In this article, we hypothesize the possibility to define specific profile of BCI users based on the contralateral activity of the ERD and the ERS. We define three BCI profiles based on accuracy: users with good accuracy i) during the KMI in the Alpha band, ii) during the KMI in the Alpha+Beta bands and iii) after the KMI in the Beta band. We also show that the accuracy is linked to the absence or presence of a contralateral activity during the observed periods.

\section{MATERIAL AND METHODS}

\section{A. Participants}

Fiftenn right-handed healthy volunteer subjects took part in this experiment (11 men and 4 women, 19 to 43 years old). They had no medical history which could have influenced the task. All experiments were carried out with the consent agreement (approved by the ethical committee of INRIA) of each participant and following the statements of the WMA declaration of Helsinki on ethical principles for medical research involving human subjects [11].

\section{B. Electrophysiological data}

EEG signals were recorded by the OpenViBE [12] platform from fiftenn right-handed healthy subjects at $256 \mathrm{~Hz}$ using a commercial REFA amplifier developed by TMS International $^{\mathrm{TM}}$. The EEG cap was fitted with 26 passive electrodes, namely $\mathrm{Fp} 1 ; \mathrm{Fp}_{\mathrm{z}} ; \mathrm{Fp} 2 ; \mathrm{F}_{\mathrm{z}} ; \mathrm{FC} 5 ; \mathrm{FC} 3 ; \mathrm{FC} 1 ; \mathrm{FC}_{\mathrm{z}}$; FC2; FC4; FC6; C5; C3; C1; $\mathrm{C}_{\mathrm{z}}$; 2 ; C4; C6; CP5; CP3;CP1; $\mathrm{CP}_{\mathrm{z}}$; $\mathrm{CP} 2 ; \mathrm{CP} 4 ; \mathrm{CP} 6$ and $\mathrm{P}_{\mathrm{z}}$, re-referenced with respect to the common average reference across all channels and placed by using the international 10-20 system positions to cover the primary sensorimotor cortex. 


\section{Protocol}

Subjects were asked to perform two different kinesthetic motor imageries to imagine the feeling of the movement (left hand and right hand). They were seated in a comfortable chair with the arms at their sides in front of a computer screen showing the cue indicated the task to perform. The whole session consisted of 4 runs containing 10 trials per task for a total of 40 trials per class.

Two panels were simultaneously displayed on the screen, which were associated from left to right, to the left hand and right hand. Each trial was randomly presented and lasted for 12 seconds, starting at second 0 with a cross at the center of each panel and an overlaid arrow indicating for the next 6 seconds the task to be performed

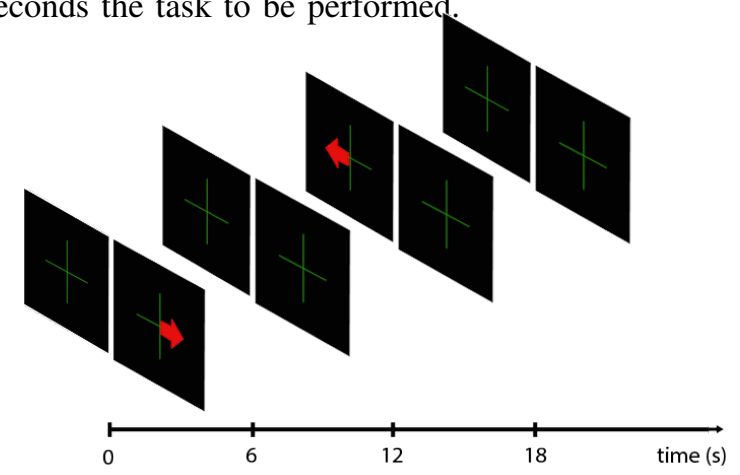

Fig. 1. Time scheme for the 2-class setup: left-hand KMI and right-hand KMI. Each trial was randomly presented and lasted for 12 second(s). During the first 6 seconds, users were asked to perform the motor imagery indicated by the task cue. The use of each body part was indicated by the presence of arrows: an arrow pointing to the left side on the left panel for a left hand $\mathrm{KMI}$, an arrow pointing to the right side on the right panel for a right hand KMI. After $6 \mathrm{~s}$, the task cue disappeared and the crosses were remaining for the next 6 seconds indicating the pause period before the next trial started.

\begin{tabular}{|c|c|c|c|}
\hline & \multicolumn{3}{|c|}{ Frequency Bands } \\
\cline { 2 - 4 } & Alpha & Beta & Alpha + Beta \\
\hline Subject & ERD [0-6 sec] & ERS [6-12 sec] & ERD [0-6 sec] \\
\hline $\mathbf{1}$ & $88,75 \pm 6,29$ & $56,25 \pm 10,31$ & $88,75 \pm 2,5$ \\
\hline $\mathbf{2}$ & $68,75 \pm 4,79$ & $83,75 \pm 6,29$ & $68,75 \pm 10,31$ \\
\hline $\mathbf{3}$ & $95 \pm 5,77$ & $58,75 \pm 2,5$ & $95 \pm 4,08$ \\
\hline $\mathbf{4}$ & $58,75 \pm 11,09$ & $73,75 \pm 4,79$ & $61,25 \pm 2,5$ \\
\hline $\mathbf{5}$ & $53,75 \pm 9,46$ & $76,25 \pm 8,54$ & $51,25 \pm 2,5$ \\
\hline $\mathbf{6}$ & $56,25 \pm 7,5$ & $68,75 \pm 4,79$ & $61,25 \pm 21,75$ \\
\hline $\mathbf{7}$ & $61,25 \pm 17,02$ & $80 \pm 7,07$ & $61,25 \pm 21,75$ \\
\hline $\mathbf{8}$ & $75 \pm 4,08$ & $61,25 \pm 14,36$ & $76,25 \pm 13,15$ \\
\hline $\mathbf{9}$ & $91,25 \pm 4,79$ & $33,75 \pm 11,09$ & $92,5 \pm 2,89$ \\
\hline $\mathbf{1 0}$ & $97,5 \pm 2,89$ & $60 \pm 5,77$ & $95 \pm 10$ \\
\hline $\mathbf{1 1}$ & $97,5 \pm 5$ & $53,75 \pm 6,29$ & $98,75 \pm 2,5$ \\
\hline $\mathbf{1 2}$ & $60 \pm 10,8$ & $73,75 \pm 6,29$ & $73,75 \pm 10,31$ \\
\hline $\mathbf{1 3}$ & $96,25 \pm 7,5$ & $42,5 \pm 2,89$ & $92,5 \pm 11,9$ \\
\hline $\mathbf{1 4}$ & $90 \pm 9,13$ & $82,5 \pm 8,66$ & $77,5 \pm 2,89$ \\
\hline $\mathbf{1 5}$ & $87,5 \pm 9,57$ & $88,75 \pm 19,31$ & $91,25 \pm 11,09$ \\
\hline AVG & $78,5 \pm 7,71$ & $66,25 \pm 7,93$ & $79,41 \pm 8,33$ \\
\hline AVG OPT & $94,58 \pm 6,51$ & $76,04 \pm 6,30$ & $90,42 \pm 6,035$ \\
\hline
\end{tabular}

Fig. 2. Accuracy results obtained by a Linear Discriminant Analysis (LDA) and using the CSP algorithm as feature extraction on the 2 classes (left-hand KMI and right-hand KMI) for 15 subjects. The classification method was applied on three frequency band (Alpha, Beta and Alpha+Beta) on the ERD time window (0-6s) and on the ERS time window (6-12s).

\section{Common Spatial Pattern}

We used the algorithm called Common Spatial Pattern (CSP) to extract motor imagery features from EEG signals; this generated a series of spatial filters that were applied to decompose multi-dimensional data into a set of uncorrelated components [13]. These filters aim to extract elements that simultaneously maximize the variance of one class, while minimizing the variance of the other one. This algorithm has been used for all conditions: the three frequency bands (Alpha, Beta and Alpha+Beta) during the ERD (0-6s) and ERS (6-12s) time windows (Figure 2).

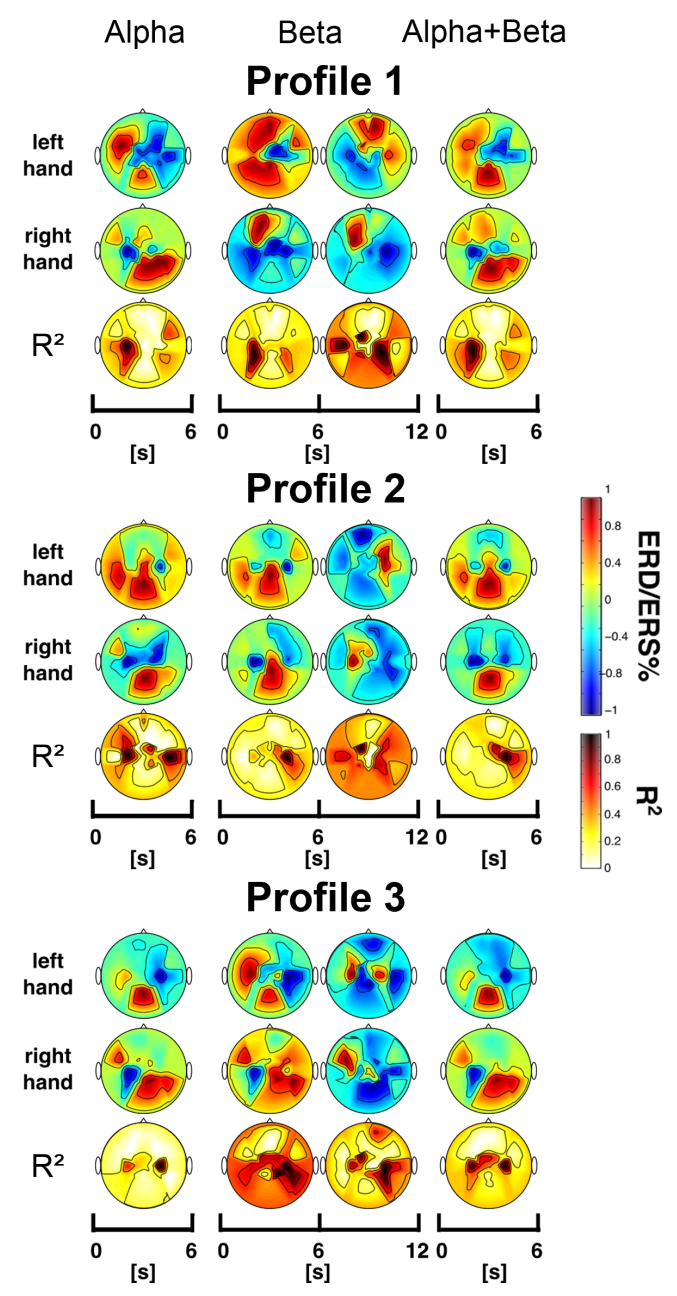

Fig. 3. Topographic map of ERD/ERS\% on three frequency bands (Alpha:7-13 Hz; Beta:15-25 Hz; Alpha+Beta:8-30 Hz) for two KMI tasks (left-hand and right-hand). Profile 1 represents grand average for Subject 10,13 and 14, who have better performance during the ERD phase (0-6 seconds) in Alpha band. Profile 2 represents grand average for Subject 2, 4, 5, 6, 7 and 12, who have better performance during the ERS phase (6-12 seconds) in Beta band. Profile 3 represents grand average for Subject 1, 3, $8,9,11$ and 15 , who have better performance during the ERD phase (0-6 seconds) in Alpha+Beta band. The red color corresponds to a strong ERS and a blue one to a strong ERD.

\section{E. ERD/ERS patterns}

To evaluate more precisely the modulation which appeared during the two different time windows, we computed the ERD/ERS\% using the "band power method" [4] with a Matlab code. First, the EEG signal was filtered considering 
one of the three different frequency bands $(7-13 \mathrm{~Hz}$, Alpha band; $15-25 \mathrm{~Hz}$, Beta band; Alpha+Beta 8-30 Hz) for all subjects using a 4th-order Butterworth band-pass filter. Then, the signal was squared for each trial and averaged over trials. Then it is smoothed using a 250 -ms sliding window with a $100 \mathrm{~ms}$ shifting step. The averaged power computed for each window was subtracted and then divided by the averaged power of a baseline corresponding to a $2 \mathrm{~s}$ window before each trial. Finally, the averaged power computed for each window was subtracted and then divided by the averaged power of a baseline corresponding $2 \mathrm{~s}$ before each trial. This transformation was multiplied by 100 to obtain percentages. This process can be summarized by the following equation:

$$
E R D / E R S \%=\frac{\overline{x^{2}}-\overline{B L^{2}}}{\overline{B L^{2}}} \times 100,
$$

where $\overline{x^{2}}$ is the average of the squared signal over all trials and samples of the studied window, $\overline{B L^{2}}$ is the mean of a baseline segment taken at the beginning of the corresponding trial, and ERD/ERS\% is the percentage of the oscillatory power estimated for each step of the sliding window. It is done for all channels separately.

ERD and ERS are difficult to observe from the EEG signal. Indeed, an EEG signal expresses the combination of activities from several neuronal sources. One of the most effective and accurate techniques used to extract events is the average technique [14]. We decided to use this technique to represent the modulation of power of the Alpha, Beta and Alpha+Beta rhythms for two KMIs tasks.

\section{RESULTS}

\section{A. Three BCI user profiles}

Table 2 shows the best accuracy obtained for each subject on a discriminative task of left-hand and right-hand KMI according to the three profiles defined in Section I. Thus, 6 subjects have a higher accuracy looking at the Beta band after the KMI, 3 subjects have a higher accuracy looking at the Alpha band during the KMI and 6 subjects have a higher accuracy looking at the Alpha+Beta band during the KMI. The best averaged accuracy over subjects were obtained considering modulations during KMI (in alpha or in alpha+beta bands). However, looking at the individual performances, we can see that 6 subjects were better considering the beta band after the KMI. For this population we can obtain a significant improvement of $13 \%$ in classification considering the activity after the KMI versus during the KMI. Using the best profile for each subject improves the averaged accuracy of $6 \%$.

\section{B. Classification rate and contralateral ERD/ERS activity}

Subjects with a higher accuracy in the Beta band after the KMI (Profile 2) have a strong ERS in contralateral during this period and a bilateral desynchronization during the KMI in the Alpha and Alpha+Beta bands (see subject 2, Fig. 4). This result is confirmed by the grand average map (Fig. 3) which shows also an ipsilateral ERD after the KMI. Finally, bilaterally ERD during the KMI, contralateral ERS and ipsilaterad ERD after the KMI could explain the high accuracy for these subjects. To validate our hypothesis, we show that the contralateral activity of subject 2 is higher for KMIs tasks on the post-KMI period in the Beta band (Fig. 5).

Conversely, subjects with a higher accuracy in the Alpha and Alpha+Beta bands during the KMI (Profiles 1 and 3) have a strong contralateral ERD during the task (Fig. 3 and Fig. 4). After the KMI, in the three frequency bands, they have no contralateral ERS or no Beta rebound on the motor cortex (see subject 10, Fig. 4). Figure 6 shows that the contralateral activity of subject 10 is higher for KMIs tasks during the KMI period in the Alpha band.



Fig. 4. Topographic map of ERD/ERS\% in three frequency bands (Alpha:7$13 \mathrm{~Hz}$; Beta:15-25 Hz; Alpha+Beta:8-30 Hz) for two KMI tasks (left hand and right hand). Subject 10 is representative of Profile 1. Subject 2 is representative of Profile 2. Subject 11 is representative of Profile 3. The red color corresponds to a strong ERS and a blue one to a strong ERD.

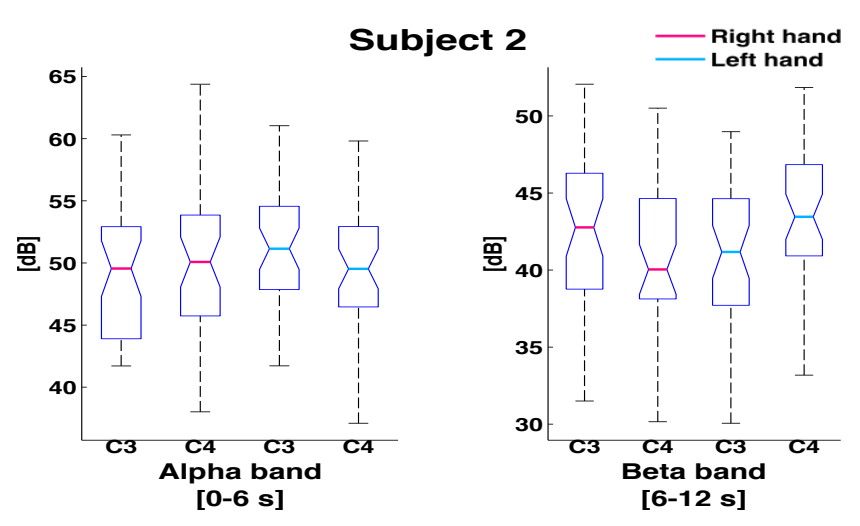

Fig. 5. Box plots of the power spectrum for Subject 2 (Profile 2) within the Alpha band and the Beta band over electrodes C3 and C4 for right hand and left hand KMIs. It can be noticed that there is a higher difference between the contralateral activity during the post-KMI period in the Beta band. 




\section{Subject 10}

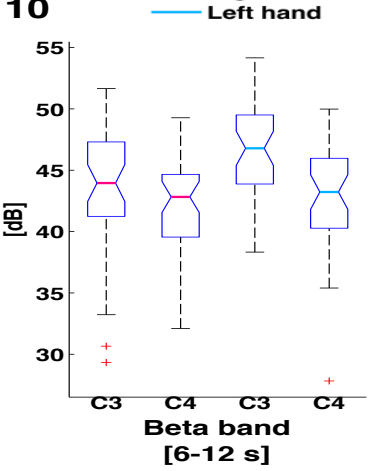

Fig. 6. Box plots of the power spectrum for Subject 10 (Profile 1) within the alpha band and the beta band over electrodes $\mathrm{C} 3$ and $\mathrm{C} 4$ for right hand and left KMIs. It can be noticed that there is a higher difference between the contralateral activity during the KMI period in the alpha band.

\section{DISCUSSION}

Subjects carried out left-hand KMIs and right-hand KMIs. Results show that 6 out of 15 subjects had a higher classification accuracy based on the post-KMI period in the beta band. This specific accuracy is due to a higher lateralization of ERD and ERS during this period.

Our study shows results which could allow to design an adaptive BCI based on contralateral activity on the motor cortex. The importance of BCI users profiles, especially for patients with severe motor impairments has already been established by other studies [15]. Moreover, it appears that there can be important changes of the contralateral activity under the choice of the frequency band [16], [17]. This is why, if we expect designing an adaptive BCI based on the specific contralateral activity of the motor cortex, it is necessary to merge these two methods.

More subjects are necessary to precise this BCI user profile. However, we investigated other KMIs (not detailed in this article), especially combined KMI (i.e. right-hand and left-hand KMI together versus right-hand KMI) and it appears that some subjects have the same BCI profile.

\section{CONCLUSIONS}

In this article, we analyzed classification accuracies to discriminate right-hand and left-hand kinesthetic motor imageries. More specifically, we distinguished two periods (i.e., during the KMI and after the KMI) for three frequency bands (Alpha, Beta and Alpha+Beta). We defined three BCI profiles based on the accuracy of 15 subjects: users with a good accuracy i) during the KMI in the alpha band, ii) during the KMI in the Alpha+Beta band and iii) after the KMI in the Beta band. This work showed that 6 out of 15 subjects had a higher classification accuracy after the KMI in the beta band, due to a contralateral ERS activity on the motor cortex. Finally, taking into account the user's lateralization profile, we obtained a significant improvement of $13 \%$ in classification for these subjects. This study show that users with a low accuracy analyzing the EEG signals during the KMI cannot be considered as BCI-illiterate. Thus, in future work, an automatic method to profile BCI users will be done allowing to design an adaptive BCI based on the best period to observe a contralateral activity on the motor cortex.

\section{REFERENCES}

[1] J. Wolpaw and E. W. Wolpaw, Eds., Brain-Computer Interfaces: Principles and Practice. Oxford university press, 2012.

[2] A. Guillot, C. Collet, V. A. Nguyen, F. Malouin, C. Richards, and J. Doyon, "Brain activity during visual versus kinesthetic imagery: an FMRI study." Hum Brain Mapp, vol. 30, no. 7, pp. 2157-2172, Jul 2009.

[3] C. Neuper, R. Scherer, M. Reiner, and G. Pfurtscheller, "Imagery of motor actions: Differential effects of kinesthetic and visualmotor mode of imagery in single-trial EEG," Cognitive Brain Research, vol. 25, no. 3, pp. 668 - 677, 2005. [Online]. Available: http://www.sciencedirect.com/science/article/pii/S0926641005002533

[4] G. Pfurtscheller and F. H. Lopes da Silva, "Event-related EEG/MEG synchronization and desynchronization: basic principles," Clin Neurophysiol, vol. 110, no. 11, pp. 1842-57, Nov 1999.

[5] G. Pfurtscheller, "Functional brain imaging based on ERD/ERS," Vision Research, vol. 41, no. 1011 , pp. $1257-1260,2001 . \quad$ [Online]. Available: http://www.sciencedirect.com/science/article/pii/S0042698900002352

[6] J. Qin, Y. Li, and A. Cichocki, ICA and Committee Machine-Based Algorithm for Cursor Control in a BCI System. Berlin, Heidelberg: Springer Berlin Heidelberg, 2005, pp. 973-978. [Online]. Available: http://dx.doi.org/10.1007/11427391·156

[7] Y. Hashimoto and J. Ushiba, "EEG-based classification of imaginary left and right foot movements using beta rebound," Clinical Neurophysiology, vol. 124, no. 11, pp. 2153 - 2160, 2013. [Online]. Available: http://www.sciencedirect.com/science/article/pii/S1388245713006457

[8] B. Z. Allison and C. Neuper, Could Anyone Use a BCI? London: Springer London, 2010, pp. 35-54. [Online]. Available: http://dx.doi.org/10.1007/978-1-84996-272-8.3

[9] G. Pfurtscheller and C. Neuper, "Motor imagery and direct braincomputer communication," Proceedings of the IEEE, vol. 89, no. 7, pp. $1123-1134$, Jul. 2001.

[10] S. Fok, R. Schwartz, M. Wronkiewicz, C. Holmes, J. Zhang, T. Somers, D. Bundy, and E. Leuthardt, "An eeg-based brain computer interface for rehabilitation and restoration of hand control following stroke using ipsilateral cortical physiology." Conf Proc IEEE Eng Med Biol Soc, vol. 2011, pp. 6277-6280, 2011.

[11] . World Medical Association, "World medical association declaration of Helsinki: ethical principles for medical research involving human subjects." J Postgrad Med, vol. 48, no. 3, pp. 206-208, Jul-Sep 2002, kIE: KIE Bib: human experimentation.

[12] Y. Renard, F. Lotte, G. Gibert, M. Congedo, E. Maby, V. Delannoy, O. Bertrand, and A. Lécuyer, "Openvibe: An open-source software platform to design, test and use brain-computer interfaces in real and virtual environments," Presence : teleoperators and virtual environments, vol. 10, no. 1, 2010.

[13] B. Blankertz, R. Tomioka, S. Lemm, M. Kawanaba, and K. Müller, "Optimizing spatial filters for robust EEG single-trial analysis [revealing tricks of the trade]," IEEE Signal processing magazine, 2008.

[14] R. Quiroga and H. Garcia, "Single-trial event-related potentials with wavelet denoising," Clinical Neurophysiology, vol. 114, no. 2, pp. 376390, 2003.

[15] J. Hohne, E. Holz, P. Staiger-Salzer, K.-R. Muller, A. Kuber, and M. Tangermann, "Motor imagery for severly motor-impaired patients: Evidence for brain-computer interfacing as superior control solution," PLOS ONE, vol. 9, 2014.

[16] K. Ang, Z. Chin, C. Wang, C. Guan, and H. Zhang, "Filter bank common spatial pattern algorithm on BCI competition IV datasets $2 \mathrm{a}$ and 2b," Frontiers in Neuroscience, vol. 6, 2012.

[17] A. Duprès, F. Cabestaing, and J. Rouillard, "Supervision of timefrequency features selection in EEG signals by a human expert for brain-computer interfacing based on motor imagery," in Systems, Man, and Cybernetics (SMC), Budapest, Hungary, Oct. 2016. [Online]. Available: https://hal.archives-ouvertes.fr/hal-01394149 